\title{
Avaliação da atenção à saúde da criança no contexto da Saúde da Família no município de Teixeiras, Minas Gerais (MG, Brasil)
}

\author{
Evaluating child healthcare in the context of Family Healthcare \\ in the city of Teixeiras, Minas Gerais (MG, Brazil)
}

\author{
Glauce Dias da Costa ${ }^{1}$ \\ Rosângela Minardi Mitre Cotta ${ }^{1}$ \\ José Roberto Reis ${ }^{2}$ \\ Maria de Lourdes da Silva Marques Ferreira ${ }^{3}$ \\ Roberta Sena Reis ${ }^{4}$ \\ Sylvia do Carmo Castro Franceschini ${ }^{1}$
}

${ }^{1}$ Departamento de Nutrição e Saúde, Universidade Federal de Viçosa. Av. PH Rolfs, s/n, Campus Universitário. 35570-000 Viçosa MG.

glaucedias@yahoo.com.br ${ }^{2}$ Departamento de Administração,

Universidade Federal de

Viçosa.

${ }^{3}$ Universidade Estadual Paulista (Unesp), campus de Botucatu.

${ }^{4}$ Graduanda, Departamento

de Nutrição e Saúde,

Universidade Federal de

Viçosa.

\begin{abstract}
The scope of this study was to analyze child healthcare in the Family Healthcare Program (FHP) in Teixeiras, MG, Brazil. A descriptive transversal study was conducted by applying a questionnaire to 161 mothers of children under 2 years of age. A specific score system was used to analyze the dimensions of structure, process and result and the respective attributes, classifying the town in the incipient, intermediary and advanced categories. The FHP was classified in the intermediary (49.6\%) category, characterized by fragmented child healthcare, though with some advances in the organization of care for this group. The physical installations, the quality of care given to control of diarrhea and respiratory infection, community participation and interdisciplinarity were considered incipient. Progress was seen in receptivity to the program, though the scant preventive and promotional activities of the FHP means that it is viewed as an annex to the hospital and merely another place for medical care. The activities of the FHP in child healthcare are not in line with the proposal for reorientation of the hegemonic healthcare model for which it was created, thereby hampering disease prevention strategies and the promotion of healthcare.
\end{abstract}

Key words Health assessment, Family health, Child health, Primary care
Resumo Este estudo tem como objetivo analisar a atenção à saúde da criança pelo Programa de Saúde da Familia (PSF) do município de Teixeiras, MG, Brasil. Foi aplicado um questionário a 161 mães de crianças menores de dois anos. Utilizou-se um sistema de escores específicos para análise das dimensões de estrutura, processo e resultado e seus respectivos atributos, classificando o município nos cenários incipiente, intermediário e avançado. Configurou-se, na sintese, um cenário intermediário (49,6\%) para o PSF, caracterizado por uma atenção à saúde da criança fragmentada, mas com avanços na organização da atenção para este grupo. Foram consideradas incipientes as instalações físicas, a qualidade do cuidado no controle da diarréia e das infecções respiratórias, a participação comunitária e a intersetorialidade. Os avanços estiveram relacionados ao acolhimento realizado pelo programa, no entanto, as atividades preventivas e promocionais do PSF demonstram que o programa surge como um suporte do hospital e mais um local de atendimento médico. A atuação do PSF na atenção a saúde das crianças distancia-se da proposta de reorientação do modelo assistencial hegemônico, pela qual foi criado, dificultando estratégias na prevenção de doenças e na promoção da saúde.

Palavras-chave Avaliação em saúde, Saúde da Família, Saúde da Criança, Atenção primária 


\section{Introdução}

A atenção à saúde da criança representa um campo prioritário dentro dos cuidados à saúde das populações. No Brasil, a ações de puericultura tiveram espaço assegurado na política de atenção a saúde da criança, entretanto, os perfis de morbimortalidade infantil resistiam às ações realizadas. De certo, as condições de saúde estavam intimamente relacionadas às condições de vida dessa população ${ }^{1}$.

Dentro deste contexto, o Ministério da Saúde adotou a AIDPI (Atenção Integrada às Doenças Prevalentes na Infância) em 1995, como política de atenção a saúde da criança menor de cinco anos. A estratégia AIDPI incorporou as ações do PAISC (Programa de Assistência Integral à Saúde da Criança), introduzindo o conceito de integralidade e propondo um novo modelo de abordagem a saúde da criança no primeiro nível de atenção (atenção básica), sistematizando o atendimento clínico e integrando ações curativas com medidas preventivas e de promoção da saúde ${ }^{2,3}$. O Programa de Saúde da Família (PSF) surge como estratégia de reorganização da atenção básica e passa a ser um importante mecanismo de mudança nas práticas e na atenção a a saúde da população.

No entanto, o cenário atual nos municípios brasileiros demonstra um desuso crescente da AIDPI. Esta afirmativa, embora ainda careça de pesquisas que a comprovem, pode decorrer de um enfraquecimento das estratégias utilizadas pelo MS para fortalecimentos das propostas da atenção. Ressalte-se também que o fato de o município utilizar a AIDPI não assegura obrigatoriamente a qualidade dos cuidados prestados à saúde da criança.

É nesse contexto que surgem indagações com relação à qualidade da atenção à saúde da criança prestada na atenção básica, aos avanços e dificuldades na operacionalização praticas e ações e por último com relação ao efeito ou impacto das estratégias para o grupo materno infantil.

Desta forma, há de se desenvolver processos avaliativos pertinentes e oportunos capazes de compreender de que forma e em que medida as ações e os princípios do PSF são direcionados à atenção à saúde da criança. Cabe refletir sobre o potencial transformador do PSF como reorganização da atenção primária e como reformulador do modelo assistencial hegemônico e biomédico tomando como medida a atenção à saúde da criança.

Paim $^{4}$ afirma que a estratégia do PSF pode assegurar a qualidade, a integralidade e a efetivi- dade do primeiro nível de atenção, ou pode visar um atendimento simplificado, tecnologicamente para a população de baixa renda. Daí, a necessidade de sua avaliação, na busca de respostas efetivas sobre seu funcionamento e sobre suas propostas de mudanças. É a partir desta proposição que se procurou situar o método utilizado neste estudo. A investigação proposta tenta, assim, se fundamentar no principio de avaliação proposto por Donabedian ${ }^{5}$ dividida em três tipos de dimensões: estrutura, processo e resultado.

O estudo tem por objetivo a avaliação da atenção à saúde da criança pelo PSF tomando como referência distintos cenários que o classifica como: cenário incipiente, cenário intermediário e um cenário avançado.

\section{Material e métodos}

A população do estudo foi composta por 161 mães de criança menores de dois anos, representando $94,7 \%$ das mães de crianças menores de dois anos do município, e por 35 (98\%) profissionais de saúde, das quatro Unidades Básicas de Saúde (UBS) do município de TeixeirasMG, constituída por quatro médicos, quatro enfermeiros, quatro auxiliares de enfermagem e 24 Agentes Comunitários de Saúde (ACS), um ACS estava de licença maternidade e não aceitou participar do estudo. O município de Teixeiras situa-se no estado de Minas Gerais, Brasil.

Trata-se de um estudo observacional de corte transversal de abordagem quanti - qualitativa em que se utiliza como instrumentos para coleta de informação questionários semi - estruturado, preparado pela equipe coordenadora, para realização das entrevistas e observações diretas realizadas pela pesquisadora.

A construção do modelo para avaliação da atenção à saúde da criança pelo PSF, foi inspirada nas proposições de Donabedian ${ }^{5}$ que relaciona as dimensões de estrutura, processo e resultado, conforme apresentado na Figura 1.

Atribuiu-se uma pontuação para cada dimensão, subdimensão e respectivos critérios, levando em consideração as relações hierárquicas entre os fatores que potencialmente contribuem para a determinação da atenção a saúde da criança (Tabela 1).

A avaliação da implantação das ações oferecidas pelo PSF ao grupo das crianças e gestantes foram medidas através de três passos. O primeiro passo foi a avaliação da estrutura, para isso um check list foi formulado com critérios adap- 


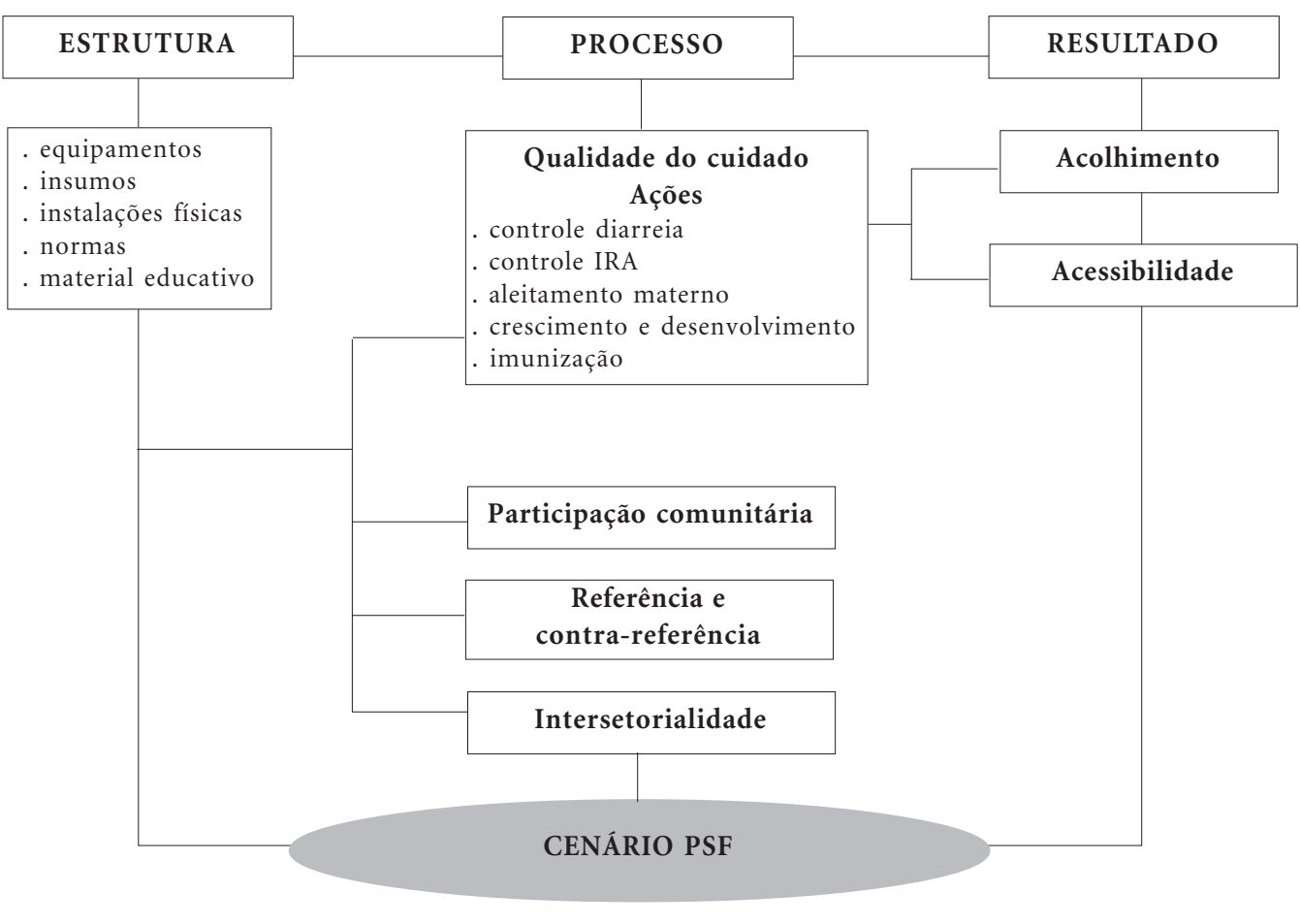

Figura 1. Desenho metodológico do estudo: atributos analisados em cada dimensão.

tados de um instrumento utilizado pela OPAS/ $\mathrm{OMS}^{6}$ - "Condições de Eficiência da Atenção da saúde da criança e da gestante", que seleciona e defini os itens da estrutura como os recursos utilizados pelo serviço na atenção à saúde da criança e analisa os seguintes atributos: (instalação física, recursos materiais e normas). Tais atributos foram classificados a partir dos critérios (sim, não e parcialmente).

$\mathrm{O}$ segundo passo diz respeito à avaliação do processo por meio de entrevistas com as mães das crianças menores de cinco anos. O processo corresponde ao conjunto de atividades desenvolvidas na relação entre profissionais e usuários. Possui como atributos a qualidade do cuidado (ações relacionadas ao controle da diarreia, das infecções respiratórias, da promoção ao aleitamento materno, do crescimento e desenvolvimento da criança e imunização), a intersetorialidade a referência e contra-referência e participação da comunidade.

O terceiro passo foi a avaliação de resultados, considerando o mesmo questionário formulado na entrevista com as mães. Os critérios desta dimensão estão relacionados com a satisfação do usuário baseada nos atributos de acolhimento (sala de espera e acolhimento) e acessibilidade (marcação de consultas e tempo de espera).

Os critérios definidos em cada dimensão (estrutura, processo e resultado) foram pontuados a fim de obter a classificação do PSF, levando em consideração as relações hierárquicas entre os fatores que potencialmente contribuíram para a determinação destes ${ }^{7-9}$. Atribui-se pesos diferenciados para cada atributo de acordo com a sua relevância para determinada ação e dimensão. Posteriormente, a dimensão de estrutura, processo e resultado e seu atributos, bem como o PSF (foi classificado como incipiente, intermediário ou avançado se obtivesse entre 0,00 e 33\%; 33,1 e $67,00 \%$ e $67,1 \%$ e $100,00 \%$, respectivamente). Os pontos de cada atributo e dimensão foram somados e depois relacionados com o máximo de pontos (em \%) para a formação do escore. Os dados foram tabulados no Excel 6.0 e SPSS 10.0 que permitiu a formação dos escores e elaboração do diagrama e das frequências.

Por fim, para verificar e analisar a atuação do PSF nas dimensões de estrutura, processo e resultado, contextualizou-se distintos cenários que 
Tabela 1. Classificação dos atributos pertencentes às dimensões de estrutura, processo e resultado - tríade da avaliação da atenção à saúde da criança do Município de Teixeiras MG.

\begin{tabular}{|c|c|c|}
\hline Atributos & Critérios & $\begin{array}{l}\text { Total máximo } \\
\text { de pontos }\end{array}$ \\
\hline \multicolumn{3}{|l|}{ Estrutura } \\
\hline Instalações físicas & $\begin{array}{l}\mathrm{N}^{\circ} \text { de salas para os profissionais; } \mathrm{n}^{\circ} \text { de banheiros; Condições } \\
\text { estruturais (iluminação, ventilação, condições das paredes e pisos); } \\
\text { local para os procedimentos - pesar, medir }\end{array}$ & 48 \\
\hline Recursos materiais & $\begin{array}{l}\text { Balança, infantometro, abaixadores de língua, termômetro, materiais } \\
\text { educativos para crianças e para as mães, soro fisiológico, } \\
\text { medicamentos, estufas, normas e procedimentos descritos para o } \\
\text { atendimento a crianças }\end{array}$ & 804 \\
\hline Total & & 852 \\
\hline \multicolumn{3}{|l|}{ Processo } \\
\hline Aleitamento & $\begin{array}{l}\text { Trabalhos educativos sobre o tema, tempo de aleitamento, } \\
\text { aleitamento exclusivo, aceitação, dificuldades }\end{array}$ & 1904 \\
\hline IRA & Presença de pneumonia, antibioticoterapia, orientações do PSF & 460 \\
\hline Diarreia & $\begin{array}{l}\text { Formas de preparo do Soro caseiro; alimentação adequada; } \\
\text { antibioticoterapia, atendimento pelo PSF }\end{array}$ & 1003 \\
\hline CD & $\begin{array}{l}\text { Frequência puericultura; acompanhamento do cartão da criança, } \\
\text { curva de crescimento e desenvolvimento }\end{array}$ & 3126 \\
\hline Imunização & Cartão de vacina; vacinas em dia & 744 \\
\hline Participação & $\begin{array}{l}\text { Participação nas atividades de promoção e prevenção - dificuldades } \\
\text { na adesão }\end{array}$ & 462 \\
\hline $\begin{array}{l}\text { Referência e } \\
\text { contra- referência }\end{array}$ & $\begin{array}{l}\text { Existência de contatos com outros níveis de atenção; consórcio } \\
\text { municipal }\end{array}$ & 16 \\
\hline Intersetorialidade & Existência de atividades desenvolvidas com as escolas, igrejas, ONGs & 70 \\
\hline Total & & 7785 \\
\hline \multicolumn{3}{|l|}{ Resultado } \\
\hline Acolhimento & $\begin{array}{l}\text { Tempo de espera; atendimento desde a recepção ate o consultório } \\
\text { dos diversos profissionais; tempo da consulta; escuta }\end{array}$ & 902 \\
\hline Acessibilidade & Facilidades ou não com transporte; marcação de consultas & 571 \\
\hline Resolubilidade & $\begin{array}{l}\text { Satisfação das mães com a atuação do PSF no atendimento as } \\
\text { crianças e a elas }\end{array}$ & 574 \\
\hline Total & & 2047 \\
\hline
\end{tabular}

foram adaptados de Copque et al. ${ }^{10}$. Em um cenário de implementação incipiente(0,00 e 33\%), a proposta aproxima-se da concepção de um programa de saúde que oferta uma atenção simplificada a saúde da criança; em um cenário intermediário (33,1 a 67\%) e o programa de saúde oferta uma atenção fragmentada a saúde da criança, mas com alguns avanços; e finalmente em um cenário avançado (67,1 a 100\%), o PSF configurar-se-ia com uma estratégia de reorganização de um dos níveis de complexidade do sistema, a atenção básica e de mudança do modelo de atenção hegemônico.

\section{Resultados e discussão}

Das mães entrevistadas, $31,7 \%$ se encontravam na faixa etária de 26-30 anos, sendo a média de idade de 27 anos $( \pm 6,1) ; 12,4 \%$ eram adolescentes e $9,9 \%$ possuíam mais de 35 anos. Eram donas de casa em sua maioria (76,5\%); 65,2\% eram casadas, $21,7 \%$ apresentaram-se em comunhão livre e $10,6 \%$ solteiras.

Com relação à renda familiar, a mediana foi de $\mathrm{R} \$ 300,00$ (um salário mínimo na época do estudo). Quanto à escolaridade, 61,5\% das mães possuíam o ensino fundamental incompleto ( $1^{\mathrm{a}}$ a $8^{\circ}$ série), sendo que destas $46 \%$ possuíam apenas de $1^{\mathrm{a}}$ a $4^{\mathrm{a}}$ série. Chegaram a concluir o ensino médio $16,1 \%$ e somente $3,1 \%$ tinham o ensino superior. 
Os profissionais de saúde eram em sua maioria do sexo feminino $74,3 \%$ e $82,9 \%$ residiam no município; $80 \%$ foram admitidos por indicação, sendo que somente $20 \%$ passaram por um processo seletivo.

\section{Análise da estrutura}

A análise da estrutura foi subdivida em instalações físicas, recursos materiais e normas existentes. As instalações físicas apresentaram-se com uma grande deficiência de espaços. Três UBS funcionam em um mesmo local, sendo que somente uma (unidade 4) possui um local próprio de funcionamento. As UBSs não possuem salas suficientes, os profissionais se alternam na realização dos atendimentos. As instalações são precárias, velhas, e com muitas dificuldades de higienização. Não têm banheiros separados para os profissionais de saúde, nem pia para higiene das mãos nas salas de atendimento. Desta forma, a análise das instalações físicas foi considerada incipiente
$(25,0 \%)$ nas unidades 1,2 e 3 e intermediária $(41,7 \%)$ na unidade 4 , revelando na medias das UBSs do município uma análise incipiente (33,3\%) (Figura 2).

Com relação aos recursos materiais, apesar da escassez, existe um aporte médio dos critérios definidos pela OPAS ${ }^{6}$ na atenção a saúde da criança, como a presença de balança pediátrica, termômetro e abaixadores de língua descartáveis, revelando uma análise intermediária $(51,7 \%)$. No entanto, há falta de medicamentos, de insumos para terapia de reidratação oral, escassez de protocolos e normas para os diferentes procedimentos relacionados às doenças prevalentes como diarreia e IRA e principalmente no que se refere a materiais educativos para controle das doenças prevalentes, para promoção do aleitamento materno, crescimento e desenvolvimento e imunização.

Copque et $\mathrm{al}^{10}$ aponta que a falta de material e medicamentos são problemas comuns nos serviços públicos de saúde e criam obstáculos ao desen-

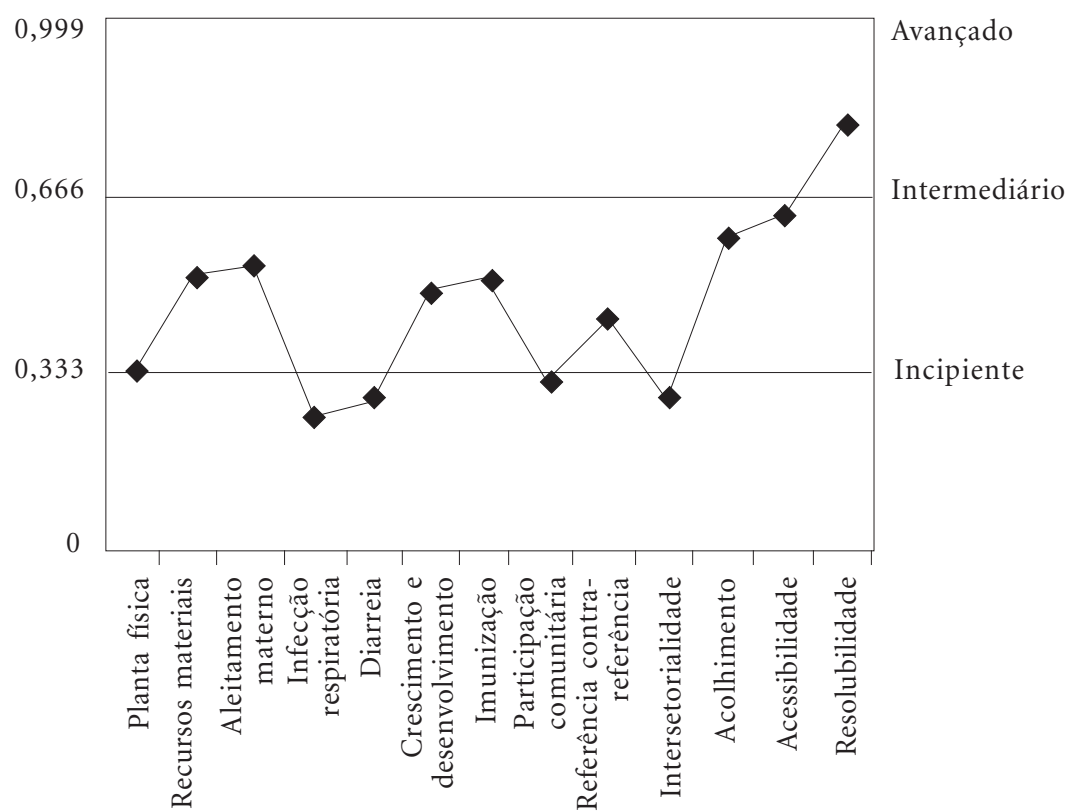

\begin{tabular}{l|l} 
Estrutura & Processo \\
Resultado
\end{tabular}

Figura 2. Classificação das dimensões de estrutura, processo e resultado e seus respectivos atributos, na atenção à saúde da criança pelo PSF - Município de Teixeiras, MG (2005-2006). 
volvimento das atividades. A insuficiência ou má distribuição dos recursos financeiros é apontada como uma dificuldade central destes problemas.

O desenho da avaliação da estrutura do PSF na atenção à saúde da criança configurou-se como intermediário $(50,7 \%)$. Ao considerar-se o espaço físico isoladamente este demonstrou-se incipiente (Figura 2).

\section{Análise do processo}

A dimensão de processo foi classificada como intermediária $(44,8 \%)$ na análise dos atributos. A participação comunitária, a intersetorialidade e a referência e contra-referência são atributos importantes que respaldam a avaliação da qualidade do cuidado (Figura 3).

A qualidade do cuidado, que foi avaliada a partir da perspectiva da mãe e dos profissionais, correspondeu uma análise das práticas de saúde direcionadas à atenção a saúde da criança pelo PSF, sendo, portanto, práticas curativas, preventivas e de promoção da saúde. Busca-se verificar o conhecimento e atuação dos profissionais, bem como a análise e perspectivas das mães frente às ações de controle da diarreia e infecções respiratórias, promoção do aleitamento, crescimento e desenvolvimento e imunização. Na Figura 4 apresenta-se a classificação de acordo com as perspectivas das mães e profissionais.

A promoção do aleitamento materno assume uma classificação intermediária na perspectiva das mães $(51,1 \%)$ e avançada $(69,1 \%)$ na perspectiva dos profissionais, conformando uma análise total intermediária (53,3\%). De acordo com o relato dos profissionais eles orientam as mães estimulando o aleitamento materno, principalmente nos seis primeiros meses de vida da criança. No entanto, alguns ACS relataram dificuldades na orientação, destacando-se a falta de capacitação, não alcançando uma porcentagem maior de adequação ${ }^{11}$.

Já na concepção das mães o aleitamento materno foi avaliado quanto ao tempo de aleitamento materno e aleitamento exclusivo até os seis meses. O tempo de aleitamento materno total para a maioria $(52,1 \%)$ das crianças chega a ser mais de quatro meses, sendo que destas 35,1\% passam dos seis meses. Em relação ao aleitamento materno exclusivo até os seis meses de idade conforme preconizado pelo $\mathrm{WHO}^{12}$, encontrou-se uma mediana de dois meses, sendo que apenas 25,6\% das crianças foram amamentadas exclusivamente até os seis meses de idade.

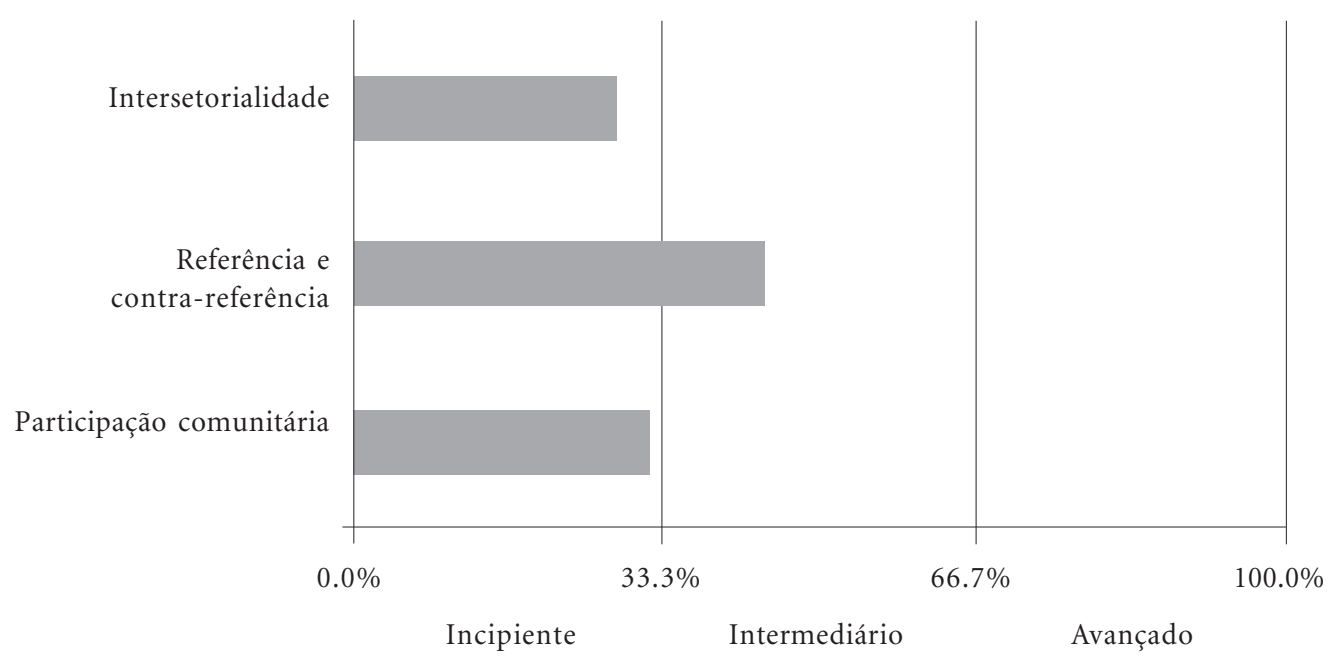

Figura 3. Classificação dos atributos intersetorialidade, referência e contra-referência e participação comunitária, pertencentes à dimensão de processo, na perspectiva das mães e dos profissionais de saúde Município de Teixeiras- MG( 2005-2006). 


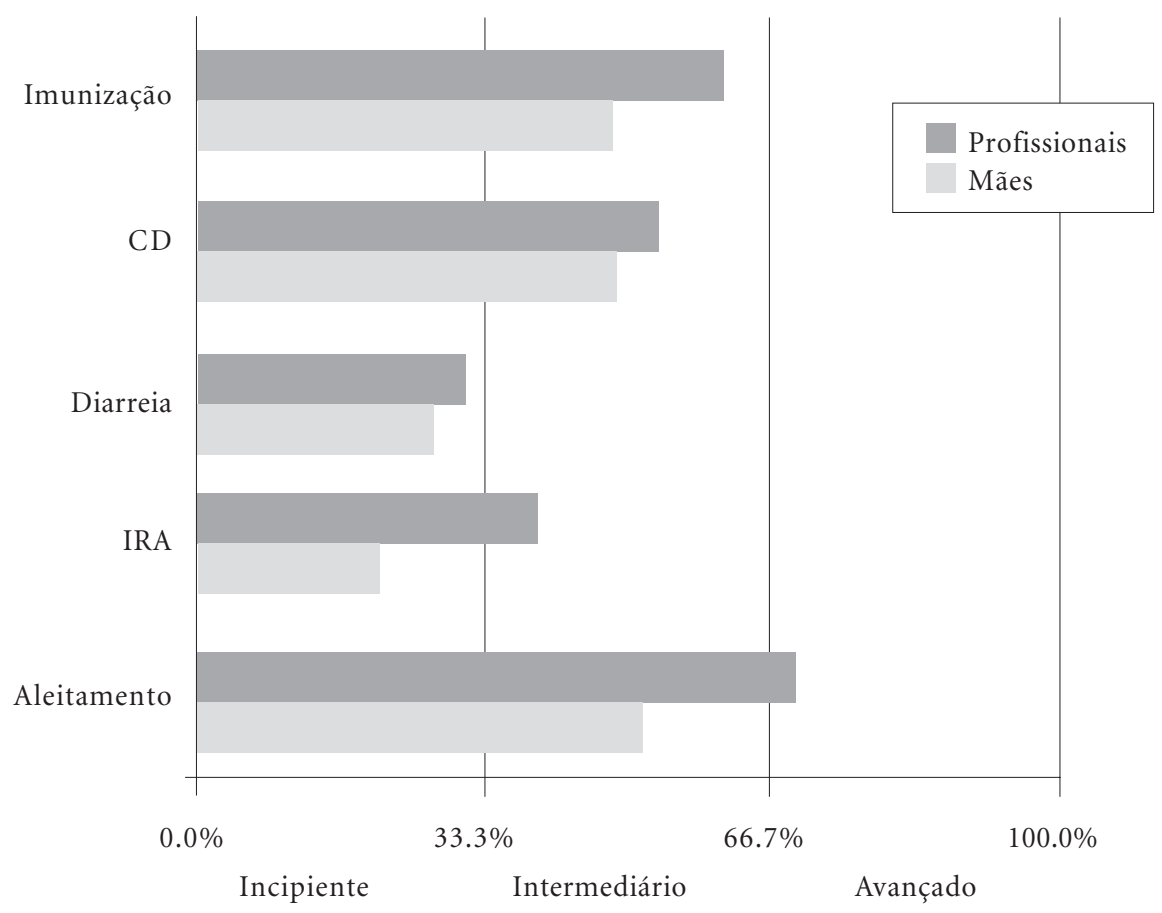

Figura 4. Classificação da qualidade do cuidado na atenção a saúde da criança pelo PSF na perspectiva das mães e dos profissionais de saúde - Município de Teixeiras- MG (2005-2006).

Fonte: Resultados da pesquisa.

O controle da diarreia, assim como da IRA, foram as ações de atenção a saúde da criança classificadas como incipientes no PSF com escores de $27,9 \%$ e 25,2\%, respectivamente. Observase que no controle da diarreia, tanto os profissionais quanto as mães configuram uma classificação incipiente, 30,6\% e 26,8\% respectivamente. Neste aspecto, foram avaliados conhecimentos específicos dos ACS como preparação do soro caseiro, diferença entre os sais de reidratação, aspectos relacionados à alimentação. Os ACS não souberam responder tais questionamentos e disseram que não foram capacitados para tal. As mães das crianças que tiveram diarreia também relataram distorções na oferta da alimentação e na oferta de líquidos. Os profissionais relataram que na UBS não realiza a Terapia de Reidratação Oral (TRO).

As infecções respiratórias foram recorrentes em várias crianças. Há falta de medicamentos específicos para as infecções como: antibióticos, antialérgicos, antigripais e inalações; falta de ati- vidades educativas para prevenção das infecções como questões simples de higienização e limpeza que ajudariam bastante no controle. Os ACS e médicos relataram a pouca ou quase inexistência de capacitação para atividades de informação e prevenção, sendo a atuação dos médicos direcionada somente às atividades clínicas.

A análise do crescimento e desenvolvimento apresentou-se com uma classificação intermediária $(48,6 \%)$. A maioria das crianças, $77,2 \%$, possui o cartão da criança, no entanto, todos os cartões, 171, estavam incompletos. Não possuem a curva de crescimento e desenvolvimento da criança no gráfico, não possuem informações sobre o peso e altura e muitas mães não compreendem o significado da curva, o cartão funciona como um cartão de vacina e não como um cartão de acompanhamento da saúde da criança. As mães relatam que os ACS não acompanham o cartão, o que é confirmado pelo número de cartões incompletos e pelos cartões perdidos que correspondem a $6,5 \%$. 
Com relação à imunização, as vacinas encontram-se em dia em $86,9 \%$ das crianças, sendo que $11,3 \%$ não estavam em dia e 1,4\% das crianças não tinham o cartão. Das crianças com cartões em atraso na vacina, as mães disseram que estavam gripadas, portanto não podiam ser vacinadas, e as que não tinham o cartão eram crianças recém nascidas. As mães relatam a não observação do cartão pelos ACS e profissionais de saúde, especialmente o médico. Vale ressaltar que este acompanhamento é essencial, visto que em alguns casos encontraram-se crianças com atrasos nas vacinas e, portanto, em risco de contágios de doenças que ofereçam risco a saúde. Os ACS relataram ser o posto de saúde do município responsável pela vacinação, mas que ainda assim informam, principalmente em época de campanhas. Nesta ação, há uma maior presença de panfletos para divulgação e informação das campanhas; sendo considerado um atributo intermediário $(51,3 \%)$.

Ao analisar as ações de atenção a criança pelo PSF caracterizada como qualidade do cuidado, que obteve uma classificação intermediária $(45,8 \%)$, verifica-se uma fragmentação especialmente nas práticas educativas. Observa-se, um despreparo dos profissionais acerca das medidas preventivas, de controle e de promoção da saúde e ainda a pouca participação dos profissionais de nível superior, que permanecem praticando apenas a orientação individual em consultório, ainda que no contexto do PSF.

Samico et $\mathrm{al}^{13}$ e Schimith ${ }^{14}$ encontraram resultados semelhantes evidenciando uma atuação mais curativa e um atendimento centrado no médico afirmando que não basta aumentar o número de equipes de Saúde da Família sem abrir espaços para novas formas de organização no processo de trabalho, entre elas destaca-se à atenção centrada no usuário, no trabalho multiprofissional e nas ações de educação, prevenção de enfermidades e promoção da saúde.

Ao se avaliar a concepção da população acerca do papel do ACS, estes atuam como "marcadores de consulta”. Marcam consultam, levam remédios, exames e formulário de visitas para a comunidade assinar, apenas alguns fazem orientações gerais sobre higiene, tratamento da água para consumo, etc.

Uma questão crucial desta análise referente aos ACS é a falta de capacitação específica para tais ações. O treinamento, a capacitação deve muni-los de conhecimentos diversos em torno da questão do processo saúde-doença, incorporando além da perspectiva biomédica, outros saberes que o habilitem neste processo de interação cotidiana com as famílias e no reconhecimento de suas necessidades ${ }^{15}$. Desta forma, o ACS assumirá a função de elo da comunidade com os serviços de saúde e de tradutor, localizando esta tradução no sentido que vai do universo cientifico ao popular.

A atuação dos profissionais de nível superior continua influenciada por práticas orientadas por um discurso biologista, que reduz a determinação do processo saúde-doença à dimensão individual. Percebe-se uma lógica predominante nos processo de saúde curativo, individual, da produção de procedimentos médicos, que vem conformando modelos assistenciais. Franco e Merhy ${ }^{16}$ revelam que os modelos configuram-se mais como produtores de procedimentos sem assumir compromissos com os usuários e suas necessidades; os procedimentos passam a ser a finalidade ultima do trabalho. Perde-se, portanto, a necessidade de se centrar a ação de saúde na lógica da produção do cuidado, ou seja, a ação que se traduz no trabalho orientado aos problemas, as necessidades e a qualidade de vida do usuário ${ }^{17}$.

Outro indicador analisado foi a participação da comunidade nas atividades desenvolvidas pelo PSF a atenção a saúde da criança, principalmente relacionadas as atividade educativas como participação em grupos, dramatizações, palestras e no próprio desenvolvimento destas. De fato, verifica-se a pouca inserção das mães nas atividades educativas, considerando este atributo como incipiente (31,6\%) (Figura 3).

Observa-se uma grande dificuldade dos profissionais de saúde do município na execução das atividades educativas para o grupo materno, as poucas iniciativas e tentativas foram frustradas pela pouca participação das mães nas atividades desenvolvidas. No entanto, 53,4\% das mães relataram a inexistência ou desconhecimento de atividades educativas no PSF, sendo que $46,6 \%$ disseram que as atividades foram realizadas com os filhos mais velhos e quando gestantes.

$\mathrm{O}$ que se pode perceber a despeito das práticas de educação em saúde é que estas atividades estão ainda fundamentadas no modelo tradicional em que os usuários são tomados como indivíduos carentes de informação em saúde, principalmente porque a pouca existência de atividades, (como por exemplo, as palestras), não contam com a participação ativa das mães no desenvolvimento destas. Desta forma, a comunicação dos profissionais de saúde assume uma atitude paternalista, no que fazer e como fazer, 
para manutenção da saúde. Paulo Freire ${ }^{18}$ revela "saber ensinar não é transferir conhecimento, mas criar possibilidades para sua própria produção ou a sua construção".

Já a referência e contra-referência foram caracterizadas por mecanismos formais de garantia de acesso à população a outros níveis de complexidade do sistema de saúde, foram considerada a existência, facilidades e dificuldades de comunicação entre os diferentes níveis de atenção. A avaliação nos aponta para uma classificação intermediária $(43,8 \%)$.

Os profissionais de saúde, médicos e enfermeiros, declararam uma maior facilidade de referência do que contra-referência. O mecanismo de organização das referências é direcionado pela secretária de saúde do município através do Consórcio Intermunicipal de Saúde, no entanto, os profissionais relatam grandes dificuldades no retorno das informações, ou seja, na contra-referencia. Não há relatório para o desenvolvimento da contra-referência, os médicos especialistas não retornam aos profissionais da atenção básica nenhum protocolo de encaminhamento, sendo que normalmente a informação é proveniente do próprio usuário. Isto ocorre, por exemplo, com a pediatra do hospital que exerce um papel importante no atendimento das crianças, mas não há nenhuma forma de organização que integre os dois serviços, dificultando a integralidade da assistência que é um dos princípios básicos do SUS.

É importante destacar que uma contra-referência adequada é a expressão mais prática de que se pode utilizar como fator de retro-alimentação extremamente vinculado às atividades do PSF $^{19,20}$.

Para efeito deste estudo a intersetorialidade foi analisada a partir de parcerias e/ou integração de outros setores nas atividades propostas pelo PSF. Desde esta perspectiva, a avaliação deste atributo foi considerada incipiente $(28,6 \%)$.

Ressalta-se que apenas as escolas do município mantêm algum tipo de parceria com o PSF e ainda assim não atendem as crianças menores de dois anos. De toda forma, a parceria estabelecida se mantêm apenas no plano burocrático, onde as escolas da zona rural "emprestam" suas salas de aula para a realização de atividades educativas, facilitando assim, o acesso das mães residentes na zona rural.

A intersetorialidade é um dos eixos estruturantes da atenção à saúde compreendendo-a como a capacidade de articular os vários setores tanto no nível mais operacional, local onde as ações são ofertadas à população, como nos ní- veis regional e central mais voltada ao planejamento e com potencialidade de articular setores fundamentais que podem desencadear mudanças para o setor saúde. Os setores como a educação, agropecuária, ambiente e habitação deveriam ser parceiros importantes na concretização das ações pensadas do ponto de vista político ${ }^{21}$. Entretanto, a intersetorialidade representa um desafio à assistência a saúde, revelado, na prática, por um modelo de atenção predominantemente biomédico. Segundo Mendes ${ }^{22}$, os sistemas fragmentados caracterizam-se por uma atenção descontínua, tendo com frequência, uma forte polarização entre um e outro ponto de atenção, em especial pela ausência de integração e ineficiência de comunicação intra e inter setorial.

\section{Análise dos resultados}

A dimensão de resultados foi analisada a partir dos atributos acessibilidade, acolhimento e resolubilidade, desde a perspectiva das mães. A classificação que se configurou ao analisar os três atributos foi intermediária $(65,9 \%)$.

A avaliação do acolhimento isoladamente resultou em uma classificação intermediária (58,5\%). Das mães que frequentam o PSF, 82,8\% se sentem bem acolhidas pelas atendentes e se sentem bem no ambiente de recepção da UBS. No discurso estão presentes fatos relacionados à educação, atenção, sorriso, simpatia e "melhor atendimento quando comparado ao hospital". Embora aspectos relacionados à organização do serviço como a falta de informação nos atendimentos médicos, tempo perdido com "filas” de espera na sala de recepção e mau humor, sejam apontados como questões negativas.

As atividades de recepção nas UBS são feitas pelas recepcionistas que seguem uma agenda com consultas diárias pré-agendadas pelos ACS, mas que não informam o horário, sendo as consultas realizadas obedecendo a ordem de chegada dos usuários. Observa-se que este é um critério de recepção excludente e criticado pelo MS no programa de humanização da atenção a saúde ${ }^{23}$. Em relação à demanda espontânea, é importante ressaltar que as mães informam preferir levar as crianças ao hospital, o que se justifica pela possibilidade de poder ser atendida por um pediatra e pela falta de vagas para demanda espontânea nas UBSs decorrente do pré- agendamento das consultas.

A atividade de recepção de um serviço de saúde deve ser entendida com um espaço em que ocorre o reconhecimento das necessidades do usuário por meio de investigação, elaboração e 
negociação ${ }^{14}$, podendo-se inferir que o encaminhamento dos usuários para o serviço de pronto-atendimento (hospital) é ditado pela organização do serviço e não pela real necessidade do usuário. Portanto, o PSF não funciona como uma porta de entrada do sistema, mas sim como mais uma opção de atendimento, o que se agrava pela predominância do modelo centrado no procedimento e na atenção médica.

Nossos achados coincidem com estudo realizado por Kovacs et al..$^{24}$ ao encontrar que os responsáveis pelas crianças não queriam ou não podiam aguardar o intervalo de tempo entre a marcação e a consulta, ou esperar na fila e não conseguir o atendimento, por isso optavam por outro serviço que não oferecido pela UBS. Cecí$\operatorname{lio}^{25}$ afirma que a rede básica de saúde não tem conseguido se tornar a "porta de entrada" mais importante para o sistema de saúde. Sendo que a porta principal continua sendo os hospitais através de seus serviços de urgência/emergência e dos seus ambulatórios.

Segundo Kovacs et al..$^{24}$ o principal motivo para as mães não levarem as crianças nas UBS, em que estavam cadastradas, é a preferência pelos profissionais da unidade de maior densidade tecnológica, devido a confiança, satisfação com o atendimento, além da acessibilidade geográfica; e termina dizendo que quando as pessoas não recebem passivamente as ações de saúde dentro de certos limites, adotam seus próprios critérios para escolher seus serviços. Desta forma, é imprescindível que os gestores e profissionais responsáveis pela saúde do município, façam questionamentos sobre as reais necessidades do ponto de vista do modelo assistencial e do usuário, encontrando alternativas mais viáveis e eficazes para a organização do acesso as atividades do PSF.

$A$ acessibilidade refere-se às características dos serviços e dos recursos de saúde que facilitam ou limitam o uso pelos usuários. Foram considerados o tempo de espera, as barreiras geográficas e a marcação de consultas como critérios de análise do atributo acessibilidade. Em relação à acessibilidade o resultado foi de um atributo intermediário $(63,0 \%)$.

A marcação das consultas é realizada pelos ACS e $62,3 \%$ das mães informaram-se satisfeitas, principalmente pela garantia de acesso e a facilidade para obtenção da consulta, já que antes do PSF era necessário "acordar de madrugada e enfrentar fila no hospital". Relatam algumas dificuldades como demora no tempo de espera para a consulta, fila e o fato do ACS só visitar uma vez por mês cada domicílio. Quanto às bar- reiras geográficas das $42,7 \%$ residentes na zona rural, $8 \%$ relataram dificuldades de acesso às consultas em períodos de chuva e falta de transporte. Apenas a UBS quatro é beneficiada com um ônibus da saúde que circula nas zonas rurais desta microárea, facilitando o acesso da população rural, as mães relataram facilidades no acesso com a presença do ônibus. O tempo de espera para o atendimento na UBS foi a maior reclamação das mães que relatam a necessidade de chegar cedo para serem atendidas com menos demora; o que pode estar justificando a procura das mães por especialista no hospital.

No que se refere à resolubilidade, foram analisados aspectos relacionados à percepção da mãe se com a implantação do PSF houve melhoria na atenção sanitária do município ou não especialmente na atenção a criança. A resolubilidade apresentou-se com a maior pontuação, configurando-se como um atributo avançado $(80,3 \%)$.

Para as mães a presença do PSF melhorou o atendimento de saúde do município, destacando-se as visitas domiciliares realizadas pelos ACS, assim como dos médicos e enfermeiros, o cuidado com os idosos, hipertensos e diabéticos, a aferição e acompanhamento da pressão arterial, a entrega de medicamentos e exames e pesagem das crianças. No entanto, percebem o PSF como mais uma opção de atendimento médico, sob os moldes do paradigma biomédico, sendo um suporte do hospital. Vale ressaltar que as mães informam que a existência do PSF ajuda a não sobrecarregar o atendimento hospitalar, não percebendo com clareza a importância do PSF nas ações de prevenção e promoção da saúde.

Neste sentido, pode-se inferir que a satisfação das mães direciona-se mais às mudanças operacionais e funcionais do que às mudanças medulares do PSF. As mudanças operacionais e funcionais se relacionam à presença do ACS que realiza suas visitas, marca consulta, leva e traz exames e medicamentos e busca ser o elo entre a comunidade e os serviços, associa-se a isto a existência da UBS que funciona como mais um local de atendimento médico. Por sua vez as mudanças medulares das práticas de saúde, ou seja, a organização e estruturação do trabalho continuam permeáveis ao modelo biomédico, não ocorrendo de fato uma mudança no entendimento da noção do sujeito que condiciona e determina os propósitos organizativos para o setor saúde ${ }^{26}$.

É evidente que as mudanças são influenciadas, por um lado por questões políticas que se preocupam mais com mudanças operacionais para fins eleitorais, algo presente e constante, 
principalmente, no interior do país e nos municípios de pequeno porte ${ }^{27}$, do que mudanças que possam interferir em rearranjos medulares exigindo tempo e informação; e por outro por questões decorrentes do próprio mercado interessado na expansão das especialidades médicas, das indústrias farmacêuticas e das tecnologias duras garantindo sua lucratividade ${ }^{16}$. De acordo com $\mathrm{Neto}^{28}$ "neste mercado da saúde frenético por lucros, a doença é vista como um potencial gerador de rendimentos".

\section{Conclusão}

A síntese das dimensões avaliadas configura-se em um cenário intermediário (49,6\%) para o PSF na atenção à saúde da criança, caracterizado por uma atenção fragmentada, mas que apresenta alguns avanços na reorganização da atenção básica e reformulação do sistema de saúde.

Os avanços estão relacionados à adesão das mães à proposta do PSF, à realização das visitas domiciliares, ao acolhimento, demonstrando uma diferenciação do atendimento hospitalar e ambulatorial tradicional e uma maior facilidade de acesso proporcionada pelo ACS.

$\mathrm{Na}$ síntese das dimensões, a falta de espaço físico interfere na realização das atividades educativas, assim como as deficiências relacionadas à participação comunitária, a intersetorialidade e a contra-referência afetam as ações de saúde desintegrando as partes e permanecendo o olhar reducionista da biomedicina. As poucas atividades preventivas e de promoção da saúde demonstram que o PSF atua em muitos momentos como um suporte do hospital e mais um local de atendimento médico, dificultando, portanto, a assimilação da integralidade em prol da reorientação do modelo assistencial.

De forma geral, percebe-se uma falta de planejamento das ações para o grupo infantil, o município segue sem atribuiçõos para este grupo oferecendo ações de cunho curativo e emergencial.

Desta forma, avaliar a atenção e a forma de organização do PSF na saúde da criança é apontar caminhos para as mudanças necessárias e capazes de promover melhorias das ações e das práticas oferecidas a este grupo. É também, apontar para a prática de um modelo de assistência hegemônico que continua influenciando a atuação dos profissionais e gestores e imobilizando a população na busca de autonomia e participação. Quiçá a divulgação destes resultados aos gestores e profissionais de saúde possibilite e gere transformações, mudanças, discussões e reorganização de suas práticas possibilitando uma maior inserção da população na formulação das atividades, rumo a reformulação do sistema de saúde hegemônico vigente.

\section{Colaboradores}

GD Costa participou na idealização, delineamento do objeto de estudo, desenho da metodologia, coleta e análise dos dados e redação do artigo; RMM Cotta participou na idealização, delineamento do objeto de estudo, desenho da metodologia, foi responsável pela coordenação, orientação e acompanhamento do trabalho de campo, redação e revisão do artigo; JR Reis participou do planejamento, da orientação técnica e acompanhamento do trabalho; MLS Marques participou na redação do artigo e sua revisão critica; RS Reis participou na elaboração do banco de dados; SCC Franceschini participou do planejamento, da orientação técnica e acompanhamento do trabalho. 


\section{Referências}

1. Prado SRLA, Fujimori E, Cianciarullo TI. A prática da integralidade em modelos assistenciais distintos: estudo de caso a partir da saúde da criança. Texto Contexto-Enferm 2007; 16(3):399-407.

2. Vidal SA, Silva EV, Oliveira MG, Siqueira AM, Felisberto E, Samico I, Cavalcante MGS. Avaliação da aplicação da estratégia da Atenção Integrada às Doenças Prevalentes da Infância (AIDPI) por agentes Comunitários de Saúde. Rev Bras Saúde Matern Infant 2003; 3(2):205-213.

3. Organização Mundial da Saúde (OMS). Avaliação global da efetividade, custo e impacto da Atenção Integrada às Doenças Prevalentes na Infância no Brasil. [acessado 2006 jun 23]. Disponível em: http//www. geocities.com/mcebrasil/apresentacao

4. Paim JS. A reforma sanitária e os modelos assistenciais. In: Rouquayrol MZ, organizador. Epidemiologia e saúde. São Paulo: Hucitec, 1999. p. 437-487.

5. Donabedian, A. Garantía y monitoria de la calidad de la atención médica: um estudo introductorio. México: Secretaria de Saúde; 1990. (Perspectivas en Salud Publica, 10).

6. OPAS. Organização Panamericana da Saúde; OMS, Organização Mundial da Saúde; Brasil/ Ministerio da Saúde. Condições de eficiência dos serviços de atenção materno-infantil. Fascículo II. 1987. (Mimeo).

7. Lopes RM, Silva LMV, Hartz ZMA. Teste de uma metodologia para avaliar a organização, acesso e qualidade técnica do cuidado na atenção à diarréia na infância. Cad. Saude Publica 2004; 20(Supl. 2): 283-297.

8. Frias PG, Lira PIC, Hartz ZMA. Avaliação da implantação de um projeto para redução da mortalidade infantil. In: Hartz ZM, Silva LMV, organizadores. Avaliação em saúde: dos modelos teóricos à pratica de programas e sistemas de saúde. Rio de Janeiro: Editora Fiocruz; 2005. p. 151-91.

9. Felisberto E, Carvalho, EF, Maggi RS, Samico I. Avaliação do processo de implantação da estratégia da Atenção Integrada às doenças prevalentes da infância no Programa Saúde da Família, no estado de Pernambuco, Brasil. Cad. Saude Publica 2002; 18(6):1737-1745.

10. Copque HLF, Trad LAB. Programa Saúde da Família: a experiência de implantação em dois Municípios da Bahia. Epidemiologia e Serviços de Saúde 2005; 14(4):223-233.

11. Pérez-Escamila, R. Patrones de la lactancia natural en la America Latina y el Caribe. Boletim of Sanit Panam 1993; 115(3):185-193.

12. World Health Organization (WHO). UNICEF (United Nations International Children's Emergency Fund). USAID (United States of America Agency International Development). SIDA (Sweden International Development Agency). Innocenti Declaration on the Protection, Promotion and Support of Breastfeeding. Florence: WHO, UNICEF; 1990.

13. Samico I, Hartz AMA, Felisberto E, Carvalho EF. Atenção à saúde da criança: uma análise do grau de implantação e da satisfação de profissionais e usuários em dois municípios do estado de Pernambuco, Brasil. Rev. Bras. Saúde Matern. Infant 2005; 5(2): 229-240.
14. Schimith MD, Lima MADS. Acolhimento e vínculo em uma equipe do Programa de Saúde da Família. Cad. Saude Publica 2004; 20(6):1487-1494.

15. Nunes MO, Trad LB, Almeida BA, Homem CR, Melo MCIC. O agente comunitário de saúde: construção da identidade desse personagem híbrido e polifônico. Cad. Saude Publica 2002; 18(6):1639-1646.

16. Franco T, Merhy E. PSF: contradições e novos desafios. [acessado 2006 maio 28]. Disponível em: http:/ /páginas.terra.com.br/saude/merhy

17. Matumoto S, Fortuna CM, Mishima SM, Pereira MJB, Domingos NAM, Supervisão de equipes no Programa de Saúde da Família: reflexões acerca do desafio da produção de cuidados. Interface - Comunic, Saúde, Educ 2005; 9(16):9-24.

18. Paulo Freire. Pedagogia da autonomia: saberes necessários à prática educativa. $30^{\circ}$ ed. São Paulo: Paz e Terra; 2004.

19. Pena Dolhun E; Grumbach K, Vranizan K; Osmond D;. Bindman, AB. Unlocking Specialists' Attitudes Toward Primary Care Gatekeepers. The journ family pract-on line. [cited 2001 Nov 16]. Disponivel em: http://www.jfponline.com

20. Machado LJC, Viana DPF, Garces AHI, Pirfo CBL, Silva MM. Interconsulta a Distância: A Utilização do e-mail na Referência e Contra-Referência de Pacientes com Doenças Endócrinas. In: Anais do $8^{\circ}$ Encontro de Extensão da UFMG; 2005 3-8. Belo Horizonte: UFMG; 2005.

21. Paula KA; Palha PF, Protti ST. Intersetorialidade: uma vivencia prática ou um desafio a ser conquistado? Interface - Comunic, Saúde, Educ 2004; 8(15): 331-348.

22. Mendes EV. A atenção primária a saúde no SUS. Fortaleza: Escola da Saúde Pública do Ceará, 2002.

23. Brasil. Ministério da Saúde. Secretaria-Executiva. Núcleo Técnico da Política Nacional de Humanização. Humaniza SUS: equipe de referência e apoio matricial. Brasília: Ministério da Saúde; 2004.

24. Kovacs MH, Feliciano KVO, Sarinho, SW, Veras AACA. Acessibilidade às ações básicas entre crianças atendidas em serviços de pronto socorro. Jornal de pediatria 2005; 81(3):251-258.

25. Cecílio LCO. Modelos tecno-assistenciais em saúde: da pirâmide ao círculo, uma possibilidade a ser explorada. In: Brasil. Ministério da Saúde. Rev-SUS, Cadernos de texto 2004; p. 93-108.

26. Carvalho SR. Saúde Coletiva e Promoção da Saúde: sujeito e mudanças; São Paulo: Editora Hucitec; 2005.

27. Cotta RMM, Mendes FF, Muniz JN. Descentralização das políticas públicas de saúde - do imaginário ao real. Viçosa: UFV, Cebes; 1998.

28. Neto OC. Dificuldades da relação médico-paciente diante das pressões do "mercado da saúde". Cien Saude Colet 2003; 8(1):307-308.

Artigo apresentado em 27/03/2008

Aprovado em 31/10/2008

Versão final apresentada em 17/01/2009 